\title{
L'enseignement de Cesarotti dans la culture européenne du jeune Foscolo : du Piano di Studj à l'Ortis
}

L'insegnamento di Cesarotti nella cultura europea del giovane Foscolo: dal Piano di Studj all'Ortis

Cesarotti's Teachings in Foscolo's European Culture: From Piano di Studj to Ultime lettere di Jacopo Ortis

Claudio Chiancone

\section{OpenEdition}

Journals

Édition électronique

URL : http://journals.openedition.org/cei/2309

DOI : 10.4000/cei.2309

ISSN : 2260-779X

Éditeur

UGA Éditions/Université Grenoble Alpes

Édition imprimée

Date de publication : 30 juin 2015

Pagination : $37-48$

ISBN : 978-2-84310-289-9

ISSN : $1770-9571$

\section{Référence électronique}

Claudio Chiancone, «L'enseignement de Cesarotti dans la culture européenne du jeune Foscolo : du Piano di Studj à l'Ortis », Cahiers d'études italiennes [En ligne], 20 | 2015, mis en ligne le 01 janvier 2017, consulté le 26 mars 2021. URL : http://journals.openedition.org/cei/2309 ; DOI : https://doi.org/ $10.4000 /$ cei.2309 


\title{
L'ENSEIGNEMENT DE CESAROTTI DANS LA CULTURE EUROPÉENNE DU JEUNE FOSCOLO : DU PIANO DI STUDJ À L'ORTIS
}

\author{
Claudio Chiancone \\ GERCI (Université Grenoble Alpes)
}

\section{L'école de Cesarotti : un magistère européiste ${ }^{\mathrm{I}}$}

C'est un fait avéré que les traductions italiennes des Poèmes d'Ossian et de l'Elegy Written in a Country Church-Yard de Thomas Gray, publiées par l'abbé padouan Melchiorre Cesarotti entre 1763 et 1772 portèrent leur auteur aux sommets de la gloire et furent surtout une source d'inspiration dans toute l'Italie ${ }^{2}$.

À partir de la fin des années I760, dans les débats et les correspondances littéraires, Cesarotti est constamment désigné comme le promoteur et le chef de file du courant sentimental et sépulcral d'inspiration nordeuropéenne ${ }^{3}$. La littérature italienne des trente années qui suivirent déborde littéralement d'opuscules de poésie mélancolique ou sépulcrale, presque toujours le fruit de poètes débutants. Parmi eux, on retrouve très souvent des échos, quand ce ne sont pas des citations extraites de l'Ossian,

I. Pour un cadre général sur Cesarotti, on se reportera à l'étude fondamentale de G. Barbarisi, G. Carnazzi (dir.), Aspetti dell'opera e della fortuna di Melchiorre Cesarotti, Milan, Cisalpino, 2002, 2 vol. Voir aussi C. Chiancone, La scuola di Cesarotti e gli esordi del giovane Foscolo, Pise, ETS, 2013; Id., "Bibliografia di Melchiorre Cesarotti», Quaderni per la Storia dell'Università di Padova, vol. XLVI, 20I3, p. 24I-272.

2. Cf. Poesie di Ossian antico poeta Celtico tradotte in prosa Inglese da J. Macpherson, e da quella trasportate in verso Italiano dall'Ab. M. Cesarotti, Padoue, Comino, 1763, 2 vol.; Elegia inglese del signor Tommaso Gray sopra un cimitero di campagna trasportata in verso italiano dall'A.M.C., Padoue, Comino, 1772.

3. Voir par exemple l'introduction à Le lamentazioni ossieno Le notti d'Odoardo Young coll'aggiunta di altre sue operette libera traduzione di Lodovico Antonio Loschi con varie annotazioni, t. I, Venise, Vitto, I774, dans laquelle le traducteur invitait Cesarotti à donner aux lettres italiennes une nouvelle traduction younguienne. Pour la diffusion de la poésie sépulcrale en Italie, cf. R. Bertazzoli, La tradizione della poesia sepolcrale e $i$ versi di Ugo Foscolo, dans G. Barbarisi, W. Spaggiari (dir.), Dei Sepolcri di Ugo Foscolo, Atti del Convegno internazionale di Gargnano del Garda (29 septembre - ${ }^{\text {er }}$ octobre 2005), Milan, Cisalpino, 2006, t. I, p. 9-62. 
mais aussi des traits élogieux, des vers, ainsi que des dédicaces au professeur padouan ${ }^{4}$. Toute une génération délaissa les poètes grecs classiques pour leur préférer les bardes (ou présumés tels) de l'Écosse antique, la poésie philosophique d'Alexander Pope, Mark Akenside et Thomas Gray, ainsi que le courant lugubre et sépulcral du Français Arnaud, des Anglais Parnell, Young et Hervey, du Suisse Haller, de l'Allemand Zachariae.

Ainsi, l'Ossian césarottien représenta non seulement une mode littéraire mais également un livre générationnel, le premier point de référence de ce "petit Sturm und Drang italien", qui, après avoir lu avec passion la Nouvelle Héloïse et pleuré devant les mésaventures du jeune Werther, était également prêt à diffuser dans la péninsule italienne les nouvelles sensibilités préromantiques 5 .

Les correspondances de Cesarotti et de son école confirment le rôle fondamental que l'abbé padouan joua dans la transmission, en Italie, de ces sensibilités nord-européennes. Ce processus se concrétisa essentiellement à travers l'œuvre réalisée conjointement avec ses élèves, que nous pouvons qualifier de cénacle césarottien. Conçu comme une famille d'élection, il s'échelonna sur trois générations successives de disciples et amis, et était rythmé par des normes qui, avec le temps, s'étaient transformées en rituels. Le Maître était le "père» de ses élèves, et ces derniers, précisément parce que «fils» d'un même père, se sentaient unis les uns aux autres par le même lien fraternel.

Entre 1779 et $\mathbf{1 7 8 2}$, au sein de la première génération de disciples, se forma une sorte de "petit cénacle» constitué de quatre disciples de Cesarotti - Francesca Roberti Franco, Pellegrino Gaudenzi, Giuseppe Urbano Pagani Cesa et Giuseppe Fossati — unis par la même passion pour les littératures européennes. Ce quatuor homogène anima une sorte de laboratoire de traductions supervisé par le Maître. C’est ainsi que la poésie française de Thomas, La Harpe et Arnaud, anglaise de Hervey, Thomson et Jerningham, ou encore allemande de Haller, Klopstock, Wieland et Schmidt fit son apparition dans les bibliothèques des Italiens cultivés ${ }^{6}$.

Lorsque la Révolution française éclata, ayant pour conséquence la politisation du débat culturel, le courant sépulcral subit un temps

4. Relevons, à titre d'exemple, l'hommage rendu par un traducteur vicentin de Klopstock à la «très belle traduction" ossianique de Cesarotti (cf. Il Messia del Signor Klopstock. Trasportato dal Tedesco in verso Italiano da Giacomo Zigno, t. I, Vicence, Modena, I782, p. v).

5. Le premier à parler de "petit Sturm und Drang italien" a été W. Binni, Preromanticismo italiano, Naples, Edizioni Scientifiche Italiane, 1959, p. 258.

6. Cf. [F. Roberti Franco, G. U. Pagani Cesa], I funerali del signor Jerningham I Sepolcri del signor Hervey e L'Eternità del signor Haller, Padoue, Conzatti [178I]; G. Fossati, Saggio di libere versioni poetiche, Padoue, Conzatti, I78I ; G. U. Pagani Cesa, Poesie, Venise, Palese, I782-I783, 2 vol. 
d'arrêt prévisible. Parallèlement à ce phénomène, on assista, à quelques exceptions près, chez les élèves de la deuxième (I785-I795) et de la troisième générations césarottiennes (I796-I808), à un affaiblissement de l'intérêt pour la littérature sépulcrale nord-européenne ainsi qu’à un repliement vers le classicisme. On commença alors à préférer le "très sage» Pope et le très classique et "divin» Gessner à un Young, un Arnaud ou un Haller

C'est précisément au moment de ce dénouement involutif qu'un nouvel aspirant «fils" fit son apparition dans l'école de Cesarotti. Le plus agité et le moins docile de tous.

\section{Européisme césarottien dans le Piano di Studj et dans les odes du jeune Foscolo}

À l'égal de l'intelligentsia grecque au sein de laquelle Cesarotti était très populaire depuis au moins une vingtaine d'années, le jeune et méconnu Niccolò Ugo Foscolo, originaire de l'île de Zante, avait vu en l'abbé padouan non seulement l'innovateur du goût à travers Ossian mais aussi et surtout un mythe littéraire et un père spirituel possible ${ }^{8}$.

Il voulut devenir le nouveau "fils" d'un tel "père», d'autant plus que, à l'époque de leurs premiers contacts (septembre I795), l'ancien professeur venait de perdre son «deuxième fils» adoré, le naturaliste Giuseppe Olivi, originaire de Chioggia.

Foscolo s'efforça alors de se faire «adopter» et de devenir le «troisième enfant» de la famille. Il entama une correspondance avec son futur «père» et ses futurs "frères ${ }^{9}$ ». D'un point de vue littéraire, cette adhésion eut des effets. Si, entre I794 et I795, la poésie foscolienne s'inspire principalement de l'hédonisme de Sappho et d'Anacréon, mais aussi de Bertola sans oublier Vittorelli, à partir de 1796 elle se tourne davantage vers le style philosophique, mélancolique et sépulcral qui était si cher à l'école césarottienne, et déjà en partie présent dans les vers élégiaques de In morte del $p_{a d r e}{ }^{\mathrm{IO}}$. Plusieurs coïncidences et rappels portent à croire que Foscolo

7. En février 1796, Greatti affirme que «le très sage Pope est le seul qui respecte à la lettre les règles du goût; et peut-être même le seul qui ait lu les anciens plus en philosophe qu'en érudit» (cf. Ep. I, p. 23).

8. Cf. Ep. I, p. I7. Dans une lettre de I799 à un destinataire non identifié, Mario Pieri définissait Corfou comme "une colonie césarottienne» (cf. Biblioteca Riccardiana di Firenze, ms. Ricc. 3546). Rappelons aussi qu'un cousin de Foscolo, Spiridione Naranzi, avait été accueilli dans le cercle des intimes de Cesarotti.

9. Cf. Ep. I, p. 17-42. On peut lire une analyse comparative de ces lettres et de celles qui circulaient habituellemet au sein du cénacle padouan dans C. Chiancone, La scuola, ouvr. cité, p. 236-237.

Io. Cf. R. Bertazzoli, La tradizione della poesia sepolcrale, art. cité, p. 32-33. Dans d'autres cas, l'anglophilie de Foscolo semble s'inspirer davantage du classicisme de Pope (voir à ce sujet E. Neppi, «Edonismo e elegia nella prima raccolta foscoliana", La Rassegna della Letteratura italiana, s. IX,, juin 200I, nº I, p. 67-68). 
entendit parler à ce moment-là du "petit cénacle» et en lit avec beaucoup d'attention les travaux.

Durant le mois de février de la même année, au cours d'une discussion épistolaire stimulante avec le bibliothécaire frioulan Giuseppe Greatti, membre de la seconde génération césarottienne, le jeune poète écrivit des «éloges des Poètes anglais» : non plus seulement Ossian et Pope, mais aussi Shakespeare et le "très passionné Young ". Ces auteurs réapparaissent sept mois après dans le fondamental Piano di Studj, à savoir dans la liste des ouvres et des lectures, faites et à faire, que le poète envoya à un autre ami intime de Cesarotti, Tommaso Olivi ${ }^{\text {II }}$. Dans le Piano di Studj, outre une "Histoire philosophique de la poésie», qui reprend à la lettre une idée lancée par le professeur padouan environ quarante ans auparavant, on retrouve tous les noms évoqués au sein de l'école césarottienne : anglais (Ossian, Gray, Milton, Pope, Young, Shakespeare, Thomson), français (Arnaud) et allemands (Gessner, Haller, Klopstock, Wieland) ${ }^{\mathrm{I2}}$.

Au cours de l'été I796, Foscolo fit un séjour à Padoue. Le même été, il rédigea deux poèmes lyriques qui constituent aujourd'hui encore le point culminant de son adhésion à l'école césarottienne, dans les deux registres, classique et sépulcral.

Dans les tercets intitulés Le rimembranze, on trouve des échos de la traduction de l'Unvollkommenes Gedicht über die Ewigkeit de Haller (I736), publiée quinze ans auparavant par Fossati sous le titre L'Eternità ${ }^{13}$, mais aussi un hommage explicite à Young, l'auteur-pivot du "petit cénacle» padouan. De toute évidence, il s'agit d'un clin d'œil aux livres de Roberti Franco et du jeune Pagani Cesa célébrant le «très sombre anglais ${ }^{14}$ ».

II. Cf. Ep. I, p. 23 ; EN II, p. 4-5. Le manuscrit du Piano di Studj est conservé à la Biblioteca Universitaria di Pavia, Autografi, 7 ; il était joint à la lettre à T. Olivi du 8 septembre 1796 (cf. Ep. I, p. 33-36).

I2. Cf. EN II, p. 6; et Opere dell'abate Melchior Cesarotti padovano, vol. XL, Pise, Capurro, I8I3, p. 55-56 (à propos du Ragionamento sopra l'origine e i progressi dell'arte poetica paru en 1762). Une excellente analyse du Piano di Studj se trouve dans U. Foscolo, Il sesto tomo dell'Io, V. Di Benedetto (éd.), Turin, Einaudi, I99I, p. $213-259$.

13. On remarquera également, dans la version de Fossati, la réflexion sur la brieveté de la vie et la référence finale à la mort, à laquelle font écho les derniers vers de l'ode foscolienne (cf. G. Fossati, Saggio di libere versioni, ouvr. cité, p. 27-36 passim).

I4. «E sul libro del duolo u' stava incisa / Eternitade e morte, a lamentarsi / Veniasi Young sul corpo di Narcisa». À noter que Foscolo avait déjà cité explicitement Young dans les vers d'occasion In morte di Amaritte (cf. R. Bertazzoli, La tradizione della poesia sepolcrale, art. cité, p. 34). Neppi a remarqué, au sujet des Rimembranze, que ces vers étaient le fruit d'une contamination entre les Night Thoughts de Young et $A$ Letter of Eloisa to Abelard de Pope, e donc d'un croisement entre les deux courants, lugubre et classique, de la poésie sépulcrale anglaise (cf. E. Neppi, Ontologia dei Sepolcri, dans G. Barbarisi, W. Spaggiari [dir.], Dei Sepolcri di Ugo Foscolo, ouvr. cité, p. IOS). 
Quant au versant plus classique de l'école césarottienne, il faut rappeler qu'une fois à Padoue, Foscolo put fréquenter Greatti en personne. Il était la figure la plus emblématique de la très modérée "seconde génération" césarottienne. On peut supposer que dans leurs conversations ils poursuivirent les discussions qu'ils avaient engagées dans leurs échanges épistolaires. Les avertissements du Frioulan concernant "ces Anglais que vous mentionnez», qui "ont le grand défaut ou d'imiter servilement, ou d'être plus extraordinaires qu'originaux", auront fait glisser la discussion $\mathrm{du}$ "très passionné Young [...] profondément enthousiaste, mais étrange parfois", aux poètes britanniques classiques du siècle précédent ${ }^{15}$.

Greatti avait traduit l'Ode to Saint Cecilia de Dryden ${ }^{16}$. Sans oublier qu'il avait été l'ami fraternel, le biographe officiel et l'éditeur posthume de Pellegrino Gaudenzi dont les débuts littéraires, avec le poème La nascita di Cristo qui s'inspirait largement du Paradise Lost de Milton, avaient rencontré un succès considérable auprès du public. Ces coïncidences expliquent l'intérêt grandissant de Foscolo pour la poésie cosmogonique, apocalyptique et biblique d'origine anglaise ${ }^{17}$.

L'école césarottienne avait repris plusieurs fois le thème miltonien de l'apostrophe au Soleil. Dans la traduction d'Ippolito Pindemonte, parue dans les Versioni dall'inglese (Venise, I794), et qui est considérée comme une des lectures cruciales du jeune Foscolo, le Satan miltonien invective ainsi : «O tu, che cinto d'alta gloria immensa / splendi là ne la tua solinga reggia / [...] e ti nomo anche, o Sole, / per dirti quanto in odio ho la tua luce $^{18}$.» Un écho de cette apostrophe miltonienne, remarqué par Fubini, est repérable dans un passage du Cartone de Cesarotti ( $\mathrm{O}$ tu celeste lampa, / dimmi, o sol, cesserai? Verrai tu manco / possente luce? $»^{19}$ ).

Le thème du contraste entre l'éternité des astres et la finitude de l'homme avait également inspiré la plume la plus prometteuse du "petit cénacle», à savoir celle de Pellegrino Gaudenzi, dans un sonnet fort intéressant. À la

15. Cf. Ep. I, p 23. On notera que le jugement de Greatti sur Young reprenait dans le fond les doutes exprimés par Cesarotti vingt-deux ans plus tôt (cf. C. Chiancone, La scuola, ouvr. cité, p. 84).

16. Cf. Versioni dall'inglese raccolte e date in luce per l'abate Angelo Dalmistro, Venise, Palese, I794.

17. Dans le Piano di Studj, Foscolo indique parmi ses «Proses originelles» une «Version du troisième livre de Milton» (cf. EN VI, p. 6). Au sujet des traductions miltoniennes de Foscolo, cf. C. Chiancone, La scuola, ouvr. cité, p. 260.

I8. Cf. Versioni dall'inglese, ouvr. cité, p. I.

19. Cf. M. Cesarotti, Le poesie di Ossian, Rome, Salerno, 200o, p. 688 ; et M. Fubini, Lettura dell' "Ortis", dans Id., Ortis e Didimo. Ricerche e interpretazioni foscoliane, Milan, Feltrinelli, 1963, p. II-85. Le passage miltonien était bien connu de Cesarotti, qui le citait dans une lettre du i8 décembre i8or (cf. Parleremo allora di cose, di persone, di libri... Lettere di Melchiorre Cesarotti a Francesco Rizzo Patarol, M. Fantato [éd.], Venise, Istituto Veneto di Scienze Lettere ed Arti, 2006, p. I6). 
mort prématurée de l'auteur, en 1784 , le sonnet suivant fut retrouvé parmi ses papiers :

\section{Al Sole}

Fonte d'eterna luce, alto sedente nell'aurea pompa di perpetui lampi, là pei deserti degli eterei campi solo il grande sei tu, solo il possente.

Una scintilla di tua faccia ardente lasci cader nel vuoto, e gli altri avvampi, ti volgi intorno in tua grandezza, e stampi il mondo di vitale orma lucente.

Vinto dal tuo splendor, prostrato a terra sacrò l'uomo al tuo Nume are e trofei, sensi d'un cor che in te s'abbaglia ed erra.

Ma te fra l'opre sue, se un Dio non sei, divinità che i doni suoi disserra, primo prescelse a ragionar di lei ${ }^{20}$.

Notons qu'au sein du "petit cénacle», Giuseppe Fossati avait aussi exploité cette thématique en écrivant une version italienne de $A$ Hymn to the Creator de Thomson (I78I) ${ }^{2 \mathrm{I}}$. Quant à Foscolo, il s'en inspira dans ses hendécasyllabes Al Sole, dont le titre reprend à la lettre le sonnet de Gaudenzi. Il y a là une sorte de défi à distance au premier élève préféré de l'école de Cesarotti, scellant l'entrée de Foscolo dans la «famille» césarottienne ${ }^{22}$. Par leur sujet d'inspiration, leur tonalité et leurs choix lexicaux, ces vers, auxquels on ajoutera ceux de La Giustizia e la Pietà (février 1797), représentent la preuve la plus tangible de l'adhésion de Foscolo à l'école padouane ${ }^{23}$.

Cette même influence se manifestera bientôt avec la même intensité dans sa prose naissante.

20. Cf. Poesie dell'abate Pellegrino Gaudenzi forlivese, Nice, Società Tipografica, I786, ad indicem. Une version manuscrite de ce poème se trouve à la BRF, ms. Ricc. 3552, f. 93.

2I. "Fonte del dì, padre del lume, o Sole, / tu che del Dio che ti creò rifulgi / l'immagine più bella, e che col tuo / foco fecondator vita diffondi / ai librati nel vuoto orbi infiniti / coi propagati tuoi raggi lucenti / sull'Universo le sue lodi imprimi» (Cf. C. Gentile, Giuseppe Luigi Fossati nella cultura veneta del suo tempo, Bari, Adda, I965, p. I34).

22. Cf. EN II, p. 3I4-316. Pour ces vers, voir aussi E. Neppi, Ontologia dei Sepolcri, art. cité, p. Io8-Io9, où l'on indique que l'explicit foscolien renoue avec les accents téléologiques et apocalyptiques de Parnell et Young. Voir également R. Bertazzoli, La tradizione della poesia sepolcrale, art. cité, p. 35-36, nous rappelant que les images de la tempête dans Al Sole semblent s'inspirer directement de La Notte de Zachariae.

23. Sur les échos younguiens et parnelliens de La Giustizia e la Pietà, cf. E. Neppi, Ontologia dei Sepolcri, art. cité, p. I06. Ce poème (écrit — rappelons-le — sur commande et remontant à février 1797, cf. C. Chiancone, La scuola, ouvr. cité, p. 273), représente, dans la poésie de Foscolo, la dernière trace d'une pleine adhésion à l'école césarottienne. 


\section{L'Ortis, entre acceptation et rupture avec le magistère césarottien}

Il a été souvent répété que la découverte de Laurence Sterne contribua à l'intérêt précoce de Foscolo pour le genre romanesque ${ }^{24}$. Mais il a été rarement indiqué que selon toute vraisemblance il le découvrit au sein l'école césarottienne.

Si l'auteur anonyme qui, en I792, publia à Venise la première traduction italienne du Sentimental Journey n'a pas encore été identifié, nous pouvons par contre affirmer avec certitude que, durant les trois années qui suivirent cette publication, le nom de Sterne circula à plusieurs reprises dans le cercle de Cesarotti. Parmi les fervents lecteurs vénitiens de l'auteur satirique anglais, on retrouve non seulement Francesca Roberti, Roberto et Giuseppe Olivi que nous avons déjà cités, mais aussi une autre figure intéressante qui gravitait autour du cénacle padouan : celle de l'écrivain originaire de Chioggia Angelo Gaetano Vianelli ${ }^{25}$.

Mais le milieu littéraire padouan s'était aussi intéressé à un autre pilier du roman sentimental nord-européen : Die Leiden des Jungen Werthers. Les deux premières éditions italiennes du roman de Goethe (I788 et I796) sont dues au médecin padouan Michiel Salom, qui était non seulement étudiant à l'université de Padoue autour de I774, mais aussi élève et admirateur des ouvres de Cesarotti, auquel il rend un hommage explicite dans une note de sa traduction, comme l'imposait la tradition de l'école ${ }^{26}$. Cela peut également expliquer pourquoi Werther est mentionné dans le Piano di Studj, qui marque l'apogée de l'adhésion de Foscolo au magistère césarottien.

C'est avec de telles prémisses, et plongé dans un tel climat culturel, que le poète de dix-huit ans décida de se consacrer à la prose intimiste et sentimentale.

Comme on le sait, on est aujourd'hui largement convaincu que la deu-

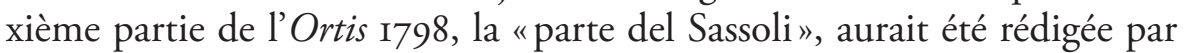

24. Cf. C. Varese, Foscolo: sternismo, tempo e persona, Ravenne, Longo, 1982, p. II-I2, et S. Parmegiani, Ugo Foscolo and English Culture, Londres, Legenda, 20II, p. 7-6o.

25. Cf. C. Chiancone, La scuola, ouvr. cité, p. 250-252. Roberti Franco fut la promotrice des Lettere di Yorick a Elisa e di Elisa a Yorick con aggiunte e note del traduttore italiano, Venise, Foglierini, 1792 (il est important de noter que l'imprimeur vénitien Foglierini était alors le libraire de confiance de Cesarotti). Quant à G. Olivi, en mars 1792 le "deuxième enfant " de la famille césarottienne demandait à l'ami Gallino une copie du Sentimental Journey et du Tristam Shandy de Sterne (cf. C. Gibin, «Lettere di Stefano Gallini a Giuseppe Olivi», Quaderni per la storia dell'Università di Padova, XXI, I988, p. I2I). Rappelons que, durant l'été 1796, Foscolo avait rédigé une ébauche d'éloge funèbre de Giuseppe Olivi.

26. Verter opera originale tedesca del celebre signor Goethe trasportata in italiano dal D.M.S., II, Venise, Rosa, I788, p. I3I. 
Foscolo lui-même et daterait probablement des années 1796-1797 ${ }^{27}$. Ce "proto-Ortis» remonterait à l'époque où Foscolo travaillait à un "certain livre», aux «Lettres à une jeune fille» et à un "Laura. — Lettere» ou bien lui serait un peu postérieur ${ }^{28}$, qui est aussi celle où il fréquentait l'école padouane ou collaborait avec elle ${ }^{29}$. Cela explique la présence de beaucoup d'autres éléments textuels et thématiques qui sont des échos évidents du milieu littéraire padouan. À part les indices les plus macroscopiques, tels l'exergue, le décor euganéen et le nom du héros, on a par exemple souligné les nombreux renvois à Ossian et à Gray, qui étaient deux textes fondateurs de l'école césarottienne ${ }^{30}$; mais on peut aussi mentionner, dans le sillage de Martelli et de Neppi, les vers que Foscolo lui-même identifie dans une note en ces termes : "Zaccaria. La notte.» Il s'agit de la traduction, faite par le jésuite vénitien Carlo Belli, d'un passage du poème Die Tageszeiten (I757) de Justus Friedrich Wilhelm Zachariae ${ }^{3 \mathrm{I}}$.

Ces vers renvoient à un milieu très proche de Cesarotti. Ils virent le jour en 1778 , lors des noces de la jeune comtesse autrichienne Leopoldina Starhemberg avec le noble Giovanni Ferro. La comtesse entama alors un dialogue long et régulier avec Césarotti, qui peut expliquer le regain d'intérêt pour la poésie allemande à partir de I779, non seulement au sein du "petit cénacle », mais dans la propre réflexion de Cesarotti ${ }^{32}$.

De même, les vers "graves et mélancoliques" que Jacopo grave sur l'écorce du cyprès auprès duquel il sera ensuite enterré, contiennent des

27. Rappelons qu'entre les mois d'avril 1797 et juin 1798, Foscolo était pris par une vie politique et journalistique des plus chargées, durant laquelle il paraît difficile qu'il ait pu se consacrer au roman naissant. Cf. M. Martelli, "La parte del Sassoli», Studi di filologia italiana, XXVII, I970, p. 177-25I; M. A Terzoli, Le prime lettere di Jacopo Ortis. Un giallo editoriale tra politica e censura, Rome, Salerno, 2004; E. Neppi, "La "parte del Sassoli" fra giallo editoriale e iperboli foscoliane di vita e di morte», Giornale storico della letteratura italiana, CLXXXIII, 2006, n 603 , p. 4I8-434.

28. Cf. EN II, p. 6, et Ep. I, p. 3I.

29. Concernant les deux séjours padouans de Foscolo (le premier entre juillet et septembre I796, le second en mars 1797) voir C. Chiancone, La scuola, ouvr. cité, p. 243-272.

30. Cf. Le quattro parti del giorno dall'originale tedesco di Federico Guglielmo Zaccaria trasportate in verso italiano dall'abate Carlo Belli, Bassano [Remondini], I778. À ce sujet, voir les analyses textuelles minutieuses de M. Martelli, "La parte del Sassoli», art. cité, et de E. Farina, Aspetti dell'ossianismo ortisiano, dans Aspetti dell'opera e della fortuna di Melchiorre Cesarotti, ouvr. cité, p. 597-6I7 (avec une large bibliographie).

3I. Cf. Ortis I798, p. 207-208 (EN IV, p. IOO). Le recours à la traduction de Belli a déjà été relevé par E. Neppi, "Il Werther e il proto-Ortis», La Rassegna della Letteratura italiana, IX, 2009, nº I, p. 34 où il est démontré que le lien entre amour, mort, et proximité de la tombe, quasiment omniprésent dans la deuxième partie de l'Ortis 1798 , découle directement des vers de Zachariae.

32. Les discussions épistolaires entamées avec le professeur hollandais Van Goens (cf. Opere dell'abate Melchior Cesarotti, ouvr. cité, vol. I, p. IOO-I6I) sont reprises, à partir de I788, dans la correspondance avec Johann Bernhard Merians de l'Académie de Berlin (ibid., vol. III, p. 58-285 passim). Leopoldina Starhemberg Ferro est citée à plusieurs reprises dans la correspondance de Cesarotti : on relèvera par exemple une lettre à Andrea Memmo, qui remonterait à 1783 et dans laquelle Cesarotti affirme être assidu "de l'incomparable comtesse Leopoldina" (ibid., vol. II, p. I85). 
traces des séjours padouans du jeune Foscolo. On y trouve par exemple des citations qui renvoient à la récente poésie émilienne. La première, qui, selon une note, serait à attribuer à "Cassiani» s'inspire en effet du poète modénais Giuliano Cassiani (I7I2-I778), tout en la modifiant pour l'adapter au désespoir amoureux de Jacopo :

Amor, mosso a pietà, sui rivi e l'erbe

Con le sue proprie mani ivi la pinge,

E la gentil mascolta, e non risponde! ${ }^{33}$

On remarquera dans le dernier vers le passage de la forme positive à la forme négative et le recours significatif au pronom "Ella», qu'on pouvait déjà «entendre " dans l'original «E la», sans oublier le point d'exclamation final, renforçant l'effet tragique du tercet.

La deuxième citation, un hendécasyllabe - «Deh più oltre veder negami amore!» — est présentée dans une note comme l'œuvre d'un "Anonyme", mais en réalité tirée du sonnet L'autore in sue nozze que le poète Angelo Mazza, originaire de Parme, avait composé en 1775 à l'occasion de son mariage avec Caterina Stocchi. Poème lyrique non seulement intime, car il fait allusion à une nuit d'amour conjugal, mais absolument inédit puisqu'il ne sera imprimé qu'en $1816^{34}$. D'où la délicate fiction de l'anonymat par laquelle le romancier protège l'identité de l'auteur. Énième ajout du Bolognais Sassoli en hommage à un très célèbre compatriote? Pas nécessairement. Les correspondances de l'époque nous révèlent que Mazza, lui-même traducteur de l'anglais et admirateur viscéral de Cesarotti depuis sa prime jeunesse, lui soumettait régulièrement ses compositions. Ses sonnets nuptiaux ne firent pas exception à la règle et avaient été envoyés au Maître le lendemain des noces. L'approbation était arrivée, ponctuellement ${ }^{35}$. On peut donc facilement supposer que ces vers furent largement diffusés au cours des années suivantes auprès des jeunes poètes de l'école césarottienne, parmi lesquels figurait Foscolo ${ }^{36}$.

33. EN IV, p. II4. Cf. Poesie scelte di Giuliano Cassiani. Edizione seconda, Vérone, I802, p. 3I : «Che amor mosso a pietà sui rivi e l'erba / Con le sue proprie man ivi la pinge, / Ella gentil m'ascolta, e mi risponde» (je souligne).

34. Cf. Opere del signor Angelo Mazza fra gli arcadi Armonide Elideo, II, Parme, Paganino, I8ı6, p. 87.

35. Cf. Opere dell'abate Melchior Cesarotti, ouvr. cité, vol. V, p. I8-20, Padoue, 9 décembre I775: "Amico carissimo / Mi congratulo colla vostra Sposa, la quale non poteva avere il più bel complimento nuziale dei vostri Sonetti»; Io février I776: «Dai vostri leggiadri e nobili Sonetti veggo che i piaceri d'Imeneo accendono in Voi più vivamente quei delle Muse; e me ne congratulo con queste vostre favorite divinità» (ibid., I, p. 260).

36. Cf. R. Bertazzoli, La tradizione della poesia sepolcrale, art. cité, p. I9, qui souligne un écho de la traduction parnellienne de Mazza dans le foscolien La Giustizia e la Pietà (I797). 
La troisième citation - "Ce n'est pas trop de toi, grand Dieu, pour la combattre ${ }^{37}$." - ramène à un contexte plus européen. La note en bas de page l'attribue à "Arnaud." Il s'agit en effet d'un vers extrait du drame Les amants malheureux ou Le comte de Comminge (1764) du poète FrançoisMarie-Thomas de Baculard d'Arnaud. ${ }^{8}$ Ce chef-d'œuvre du drame noir avait fait l'objet en France de nombreuses rééditions et ce vers en particulier avait joui d'une certaine popularité en Italie grâce à un autre poète bolognais, Francesco Albergati Capacelli. Ce dernier, imprimant une traduction italienne du drame (I78I), avait rendu de la manière suivante les imprécations du protagoniste, rendu furieux par son amour impossible pour la belle Adélaïde :

Ho l'inferno nel cor: me non conosco.

Armati pur, o Dio vendicatore,

Contro un nemico, ch'amo, e che idolatro.

Gran Dio, a pugnar con lui tu basti appena.

Le dernier vers ayant paru trop obscur au traducteur, celui-ci, dans une note, avait reproduit l'original, accompagné d'une explication : "Ce n'est pas trop de toi, grand Dieu, pour le combattre. / Expression emphatique d'un homme hors de sens ${ }^{39}$."

Ici aussi, l'origine émilienne du vers ne doit pas nous induire en erreur. À l'instar d'Angelo Mazza, Francesco Albergati Capacelli fut le correspondant, le confident et l'hôte de Cesarotti pendant près de quarante ans. De plus, Albergati était l'un des auteurs de prédilection du "petit cénacle», et Pagani Cesa qui lui avait dédié ses premières traductions ${ }^{40}$. Le vers d'Arnaud est un autre écho du milieu littéraire césarottien dans les pages du jeune Foscolo.

\section{Ortis 1798 et Ortis 1802 : derniers échos césarottiens}

Au lendemain de la chute de la République vénitienne, le ralliement enthousiaste de Foscolo aux idéaux révolutionnaires produisit dans sa vie

37. EN IV, p. II4 (souligné par Foscolo).

38. Une édition de ses "Drammi» sera également retrouvée sur la table de Jacopo après sa mort.

39. Cf. Il conte di Commingio. Tragedia di M.r d'Arnò tradotta in italiano dal marchese Albergati Capacelli, Naples, Vinaccia, I78I, p. 36 (je souligne).

40. Cf. G. U. Pagani Cesa, Saggio delle poesie pastorali del Sig. Gesnero, Belluno, Tissi, I779. La correspondance entre Cesarotti et Albergati Capacelli, encore en partie inédite, avait commencé en 1765 , à l'époque où le Padouan était précepteur à Venise. Comme je l'ai montré ailleurs, Cesarotti fut hôte d'Albergati à Bologne au moins à deux reprises, en I79I et I793 (cf. C. Chiancone, La scuola, ouvr. cité, p. 55 et IIO). 
une rupture, qui entraîna une prise de distance à l'égard du Maître. L'élaboration du roman la révèle. Dans Ortis 1802 on constate la disparition d'échos césarottiens qui étaient présents dans les versions précédentes du roman. Ceux qui restent changent de nature, et constituent pour la plupart des références subliminales à une époque désormais révolue.

Avec Ortis, Foscolo a choisi un modèle qui demeure certes, européen, marqué toutefois par un radicalisme et par un nihilisme allant bien audelà de l'Elegy césarottienne et des autres traductions du petit cénacle. Dans Ortis I802 il n'est plus question de la douce mélancolie d'Ossian, ni du sentiment religieux de Young. Werther, de par son côté "venimeux" était du reste une lecture que Cesarotti interdisait à ses disciples ${ }^{41}$.

L'éloignement de l'école césarottienne se fait aussi sentir sur le plan du style et du contenu. Le nombre de références à Ossian et à Gray diminue sensiblement ${ }^{42}$. Le long remaniement de l'Elegy césarottienne qui clôturait la lettre du ro mai disparait ${ }^{43}$. La lecture de «certains chants d'Ossian» que Lorenzo faisait à Jacopo est également rayée ${ }^{44}$. Non seulement les onze vers de l'Ossian césarottien sont éliminés mais aussi cette espèce de centon ossianique chanté à la harpe par Thérèse ${ }^{45}$. De façon encore plus emblématique, Ossian est écarté de la triade des grands auteurs de la littérature mondiale (Homère, Ossian et Dante) et remplacé par Shakespeare ${ }^{46}$. Le Maître lui-même, le "Poète de la Nation », le «Père» des lettres de I795-I796 devient le semi-anonyme "Professeur $\mathrm{C}^{* * *}$ » en opposition aux habitudes de l'école césarottienne au sein de laquelle l'hommage explicite au Maître était coutumier. L'école césarottienne n'est plus présente que par de vagues allusions. On pourrait les interpréter comme un dernier et tardif hommage à un groupe qui lui avait ouvert tant de voies.

Derrière la brève parabole humaine du personnage $\mathrm{d}^{\text {' }}$ Olivo $\mathrm{P}^{* * *}$ ", orphelin de père et innocente victime de sa propre ingénuité, on peut ainsi reconnaître les mésaventures conjointes de Pier Antonio Bondioli et de Giuseppe Olivi, respectivement "premier enfant» et "deuxième enfant» de la famille césarottienne. Tous deux disparus prématurément ${ }^{47}$. Derrière

4I. La citation est tirée de la célèbre lettre du 30 avril I803 de Cesarotti à Barbieri (cf. Opere dell'abate Melchior Cesarotti, ouvr. cité, vol. V, p. 8).

42. À noter, à ce sujet, les considérations de E. Farina, Aspetti dell'ossianismo ortisiano, art. cité, p. 608.

43. Cf. EN IV, p. 55-56.

44. Cf. EN IV, p. IO7.

45. Le premier à avoir remarqué que la chansonnette que Thérèse chante en jouant de la harpe était un centon ossianique (cf. EN IV, p. 80-83) a été M. Martelli, «La parte del Sassoli», art. cité, p. 235-237.

46. Cf. EN IV, p. 59 et 36 I.

47. Comme j'ai soutenu ailleurs, le discours vibrant de Jacopo en défense de la mémoire de Olivo P*** est fort probablement une réutilisation de l'éloge funèbre de Giuseppe Olivi, que Foscolo avait promis au frère 
les anciens disciples devenus "gentilshommes des îles déjà vénitiennes», à qui le «Professeur $\mathrm{C}^{* * *}$ " adresse des lettres qui ne parviendront jamais à leurs destinataires à la fin du roman, on peut lire une allusion aux élèves grecs de Cesarotti : Bondioli, les deux Naranzi, Mario Pieri et Foscolo luimême ${ }^{48}$. Nous assistons ici à un passage de témoin raté, à la prise de congé d'une famille où le jeune poète avait tant espéré être accueilli. Renoncement à une adoption qui n'avait jamais eu lieu. Une expérience révolue mais que Foscolo aura voulu inscrire à jamais dans le «livre de son cœur ${ }^{49}$ ».

du défunt le 8 septembre 1796, mais qui était resté à l'état d'ébauche (cf. Ep. I, p. 35; C. Chiancone, La scuola, ouvr. cité, p. 294-302).

48. Cf. EN IV, p. 464.

49. Foscolo décrit ainsi son roman dans la célèbre lettre à Cesarotti du II septembre I8O2. 